\title{
Evaluadores de este número
}

Alicia Anaveda (Universidad Nacional de San Juan)

Rocío Canudas (Universidad Nacional Autónoma de México e INDES-BID)

Diego Ceruso ( Universidad de Buenos Aires)

Maria Eugenia Comerci (Universidad Nacional de La Pampa-CONICET)

Francisco Lavolpe (Universidad Nacional de Lomas de Zamora)

Patricia Lucero (Universidad Nacional de Mar del Plata)

Luciano Visentini (Universidad degli studi di Firenze, Italia) 